\title{
Quadrupole Contribution to Two Neutron Removal in Heavy Ion Collisions
}

\author{
C.J. Benesh, \\ T-5, MS B283 Los Alamos National Laboratory \\ Los Alamos, NM 87545
}

July 11, 2018

\begin{abstract}
In this report, electric quadrupole corrections to the two neutron removal cross section measured in heavy ion collisions are estimated for ${ }^{197} \mathrm{Au}$ and ${ }^{59} \mathrm{Co}$ targets. The quadrupole process is assumed to proceed primarily through excitation of the giant isovector quadrupole resonance, which then decays by neutron emission. For ${ }^{59} \mathrm{Co}$, the contribution from E2 radiation is found to be small, while for ${ }^{197} \mathrm{Au}$ we find the quadrupole contribution resolves the discrepancy between experiment and the simple predictions of the Weissacker-Williams virtual photon method.
\end{abstract}

The combination of large charges and enhancement of the radiation pulse at high energies by Lorentz contraction make Electromagnetic Dissociation(ED) by heavy ions an attractive method for measuring low energy nuclear cross sections of astrophysical interest [1]. At the same time, the very large cross sections, on the order of barns, attainable in ED will contribute substantially to the hadronic background at RHIC[2]. A thorough understanding of 
the ED process is necessary to extract meaningful results from these experiments. It is not clear, however, that such an understanding exists at present. Indeed, it has been found in target fragmentation experiments that the single and double neutron removal cross sections do not agree with the simple Weissacker-Williams(WW) theory of the ED reaction [2, 过, 田, 5, 旬. For the most part, the discrepancies in the single neutron removal process have been resolved by careful consideration of the choice of critical impact parameter [7], inclusion of quadrupole excitations in the EM cross section[7], and the effect of the Rutherford bending of the projectile's trajectory $[\mathbb{} \|$. Recently, these considerations have begun to be applied to the two neutron removal process as well[9]. In this report, we examine the role played by quadrupole excitations, in particular, the Isovector Giant Quadrupole Resonance(IVGQR), in the two neutron removal process.

To date, the most thorough study of the systematics of the two neutron removal process have been carried out by the Iowa State Group[2]. For a variety of projectiles, they have measured the two neutron removal cross sections on ${ }^{197} \mathrm{Au}$ and ${ }^{59} \mathrm{Co}$ targets(see table). Using factorization to to estimate the piece of the cross section due to nuclear processes, they extract the electromagnetic contribution and find sizeable discrepancies from the predictions of the simple WW calculation for the Au target.

In reference 9, Norbury advances the idea that the discrepancies may be understood if one assumes that the uncertainties in the experimental mea- 
surements have been underestimated. As evidence for this, he assumes that the ratio of the experimental cross sections for one and two neutron removal should be independent of the projectile, and then estimates a revised "experimental" cross section for ${ }^{197} \mathrm{Au}$ targets using the ${ }^{56} \mathrm{Fe}$ data point, which agrees with the simple WW calculation, to normalize the remaining data. The revised cross sections agree to within a few millibarns with the naive WW prediction. Underlying this procedure is the assumption that the one and two neutron removal cross sections are both dominated by the naive WW cross sections, which do have an approximately constant ratio. For single neutron removal, this assumption is reasonably valid, as the corrections due to quadrupole excitations are on the order of ten per cent for the projectiles under consideration[7]. This is most likely not the case for two neutron removal. Unlike the single neutron case, the two neutron threshold(15 MeV for $\left.{ }^{197} \mathrm{Au}\right)$ lies above the energy of the Giant Dipole Resonance(GDR)(13.8 $\mathrm{MeV}$ for ${ }^{197} \mathrm{Au}$ ), so that contribution of the GDR is considerably smaller than it is for single neutron removal. On the other hand, the isovector giant quadrupole resonance lies above the two neutron threshold(23 MeV for Au) and as a result decays primarily into two neutrons. In addition, for the lowest energy projectiles, where the discrepancy is largest, the WW quadrupole flux will be enhanced relative to the dipole flux. Thus, a reasonable expectation is that the quadrupole contribution will be a significantly larger percentage of the two neutron cross section than for single neutron removal. 
In order to estimate this contribution, one needs a model to separate the quadrupole part of the photo-cross section from the dipole piece. The quadrupole cross section is assumed to dominated by the IVGQR, with a correction factor for the two neutron threshold,

$$
\sigma_{E 2}(E)=\frac{f \sigma_{E W S R} E_{I V G Q R}^{2}}{\left(1+\left(E^{2}-E_{I V G Q R}^{2}\right)^{2} / E^{2} \Gamma^{2}\right)} \frac{\left(E-E_{t h}\right)^{2}}{\left(\Lambda^{2}+\left(E-E_{t h}\right)^{2}\right)},
$$

where $f$ is product of the fractional saturation of the energy weighted sum rule and the branching ration for two neutron decay, $E_{I V G Q R}$ is the resonance energy, $\Gamma$ the resonance width, $E_{t h}$ the two neutron removal threshhold, $\sigma_{E W S R}$ is the energy weighted sum rule[9], and $\Lambda$ a parameter which determines how rapidly the two neutron channel opens. From reference 11 and statistical model calculations [12], $f \approx .8, E_{I V G Q R}=23 \mathrm{MeV}$, and $\Gamma=7$ $\mathrm{MeV}$ for ${ }^{197} \mathrm{Au}$. For ${ }^{59} \mathrm{Co}$, there is no data on the IVGQR available, so the parameters for ${ }^{58} \mathrm{Ni}$ are used( $\left.f \approx .1, E_{I V G Q R}=29 \mathrm{MeV}, \Gamma=9 \mathrm{MeV}\right)$ The parameter $\Lambda$ is determined from photonuclear data to be roughly $2 \mathrm{MeV}$ for both $\mathrm{Co}$ and $\mathrm{Au}$ targets.

The ED cross section is determined by folding the equivalent photon fluxes [1] over the cross sections. Assuming that only the first two electric multipoles are important,

$$
\begin{aligned}
\sigma_{E D} & =\int_{E_{t h}} d E \sigma_{E 1}(E) n_{E 1}(E)+\int_{E_{t h}} d E \sigma_{E 2}(E) n_{E 2}(E) \\
& \approx \int_{E_{t h}} d E \sigma_{p h o t o}(E) n_{E 1}(E)+\int_{E_{t h}} d E \sigma_{E 2}(E)\left(n_{E 2}(E)-n_{E 1}(E)\right),
\end{aligned}
$$


where $\sigma_{\text {photo }}(E)$ is the experimental photoneutron cross section, and $n_{E n}$ is the appropriate photon flux for the nth multipole from reference 1. The

resulting cross sections are shown, along with the experimental results and the E2 correction, in the table below.

For Co, the additional contribution from the quadrupole flux is mitigated by the lack of IVGQR strength, and the already good agreement between theory and experiment remains undisturbed. For the Au target, the ED cross section is increased by roughly a third for all projectiles and agreement with the experimental results is improved for all projectiles except Fe, which, as noted in reference 9, lies suspiciously low relative to the other data. The remaining discrepancies are for cases were the measured ED cross section is small, and consequently more sensitive to possible systematic errors in measurement and/or extraction of the nuclear contribution. Thus, one may conclude that the discrepancies observed in the two neutron ED cross sections may be well described by the WW method if the E2 strength present in the IVGQR resonance is properly accounted for.

\section{Acknowledgements}

This work was supported in part by the U.S. Department of Energy, Division of High Energy and Nuclear Physics, ER-23.

\section{References}


[1] C.A. Bertulani and G. Baur, Phys. Rep. 163, 300(1988), G. Baur, C.A. Bertulani, and H. Rebel, Nucl. Phys. A458, 188(1986).

[2] J.C. Hill, F.K. Wohn, J.A. Winger, M. Khayat, M.T. Mercier, and A.R. Smith, Phys. Rev. C39, 524(1989).

[3] M.T. Mercier, J.C. Hill, F.K. Wohn, and A.R. Smith, Phys. Rev. Lett.

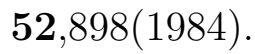

[4] M.T. Mercier, J.C. Hill, F.K. Wohn, C.M. McCullough, M.E. Nieland, J.A. Winger, C.B. Howard, S. Renwick, D.K. Matheis, and A.R. Smith, Phys. Rev. C33,1655(1986).

[5] J.C. Hill, F.K. Wohn, J.A. Winger, and A.R. Smith, Phys. Rev. Lett. 60,999(1988).

[6] A.R. Smith, J.C. Hill, J.A. Winger, and P.J. Karol, Phys. Rev. C38,210(1988).

[7] C.J. Benesh, B.C. Cook, and J.P. Vary, Phys. Rev. C40, 1198(1989), J.W. Norbury, Phys. Rev. C40, 2621,(1989), J.W. Norbury and L.W. Townsend, Phys. Rev. C42, 1775,(1990), J.W. Norbury, Phys. Rev. C41, 372,(1990), J.W. Norbury, Phys. Rev. C42, 711,(1990), J.W. Norbury, Phys. Rev. C42, 2259,(1990).

[8] A.N. Aleixo and C.A. Bertulani, Nucl. Phys. A505,448(1982). 
[9] W.J. Llope and P. Braun-Munzinger, Phys. Rev. C41, 2644(1990), J.W. Norbury, Phys. Rev. C45, 3024(1992).

[10] A.S. Goldhaber and H.H. Heckman, Ann. Rev. Nuc. Part. Sci. 28, 161(1978), R.M. Raisbeck and F. Yiou, Phys. Rev. Lett. 35, 155(1975), D.L. Olson, B.L. Berman, D.E. Greiner, H.H. Heckman, P.J. Lindstrom, and H.J. Crawford, Phys. Rev. C28, 1602(1983).

[11] F.E. Bertrand, Ann. Rev. Nucl. Sci. 26,457(1975).

[12] M. B. Chadwick, private communication.

[13] D.D. Schwellenbach, J.C. Hill, F.K. Wohn, P.R. Graham, C.J. Benesh, A.R. Smith, D.L. Hurley and P.J. Karol, "Electromagnetic Dissociation of ${ }^{59} \mathrm{Co}$ and ${ }^{197} \mathrm{Au}$ targets by relativistic ${ }^{238} \mathrm{U}$ projectiles", in preparation. 


\begin{tabular}{|c|c|c|c|c|c|c|}
\hline$\underline{\text { Projectile }}$ & $\underline{\text { Target }}$ & $\underline{\text { Energy }}$ & $\sigma_{E M}^{e x p t}$ & $\sigma_{E M}^{w w}$ & $\delta \sigma_{E 2}^{w w}$ & $\sigma_{E M}^{W W}+\delta \sigma_{E 2}$ \\
\hline${ }^{12} \mathrm{C}$ & ${ }^{59} \mathrm{Co}$ & 2.1 & $6 \pm 4$ & 1.1 & 0.05 & 1.2 \\
\hline${ }^{20} \mathrm{Ne}$ & ${ }^{59} \mathrm{Co}$ & 2.1 & $3 \pm 5$ & 2.9 & 0.1 & 3.0 \\
\hline${ }^{56} \mathrm{Fe}$ & ${ }^{59} \mathrm{Co}$ & 1.7 & $13 \pm 6$ & 14 & 0.6 & 14.6 \\
\hline${ }^{139} \mathrm{La}$ & ${ }^{59} \mathrm{Co}$ & 1.26 & $32 \pm 16$ & 44 & 2 & 46 \\
\hline${ }^{238} \mathrm{U}$ & ${ }^{59} \mathrm{Co}$ & 0.96 & $80 \pm 24$ & 65 & 4 & 69 \\
\hline${ }^{12} \mathrm{C}$ & ${ }^{197} A u$ & 2.1 & $9 \pm 17$ & 5 & 2 & 7 \\
\hline${ }^{20} \mathrm{Ne}$ & ${ }^{197} A u$ & 2.1 & $49 \pm 15$ & 14 & 5 & 19 \\
\hline${ }^{40} \mathrm{Ar}$ & ${ }^{197} A u$ & 1.8 & $76 \pm 18$ & 38 & 13 & 51 \\
\hline${ }^{56} \mathrm{Fe}$ & ${ }^{197} A u$ & 1.7 & $73 \pm 13$ & 73 & 25 & 98 \\
\hline${ }^{139} L a$ & ${ }^{197} A u$ & 1.26 & $335 \pm 49$ & 238 & 86 & 324 \\
\hline${ }^{238} \mathrm{U}$ & ${ }^{197} A u$ & 0.96 & $470 \pm 110$ & 430 & 173 & 603 \\
\hline
\end{tabular}

Two neutron removal cross sections for ${ }^{59} \mathrm{Co}$ and ${ }^{197} \mathrm{Au}$ targets $\sigma_{E M}^{\text {expt }}$ from reference $11,{ }^{238} U$ from reference $13 . \delta \sigma_{E 2}^{w w}$ is the correction to the naive Weissacker-William cross section due to the isovector E2 resonance. 